\title{
Generalized Bronchiectasis Due to Extensive Deficiency of Bronchial Cartilage
}

\author{
H. E. WILLIAMS, L. I. LANDAU, and P. D. PHELAN \\ From the Clinical Research Unit, Royal Children's Hospital Research Foundation, Royal Children's Hospital, \\ Melbourne, Australia
}

Williams, H. E., Landau, L. I., and Phelan, P. D. (1972). Archives of Disease in Childhood, 47, 423. Generalized bronchiectasis due to extensive deficiency of bronchial cartilage. The clinical, radiological, and physiological findings in 16 patients with extensive bronchiectasis due to deficiency in bronchial cartilage are discussed. These features do not occur in other types of bronchiectasis and comprise a distinctive syndrome.

The main clinical features are persistent cough and wheezy breathing. The symptoms are first noticed in infancy often after mild respiratory infection, but at times after measles or pneumonia. Most of the children develop chest deformity and clubbed fingers, and are small in stature.

The radiological findings are pulmonary hyperinflation, air-filled dilated bronchi, and, bronchographically, the bronchi balloon during inspiration and collapse with expiration.

Physiologically the outstanding features are gross increase in residual lung volume, severe impairment of maximum expiratory flow rates, especially at low lung volumes, a marked decrease in conductance in the upstream segment, poor static elastic recoil, and dynamic compliance which is frequency dependent.

It is postulated that this syndrome is due primarily to extensive developmental deficiency of bronchial cartilage, with secondary recurrent respiratory infection.

In 1960, Williams and Campbell reported 5 patients with a very unusual pattern of generalized bronchiectasis, the outstanding features being soft, compliant bronchi which dilated and collapsed during inspiration and expiration. 2 of the 5 children died, and necropsy studies in one of them showed that there was extensive deficiency of the cartilage from the fourth to the sixth or eighth generation bronchi.* It was considered that the cartilage deficiency was due to failure of development, and not the result of destructive changes after inflammation from either bacterial or viral infection.

This syndrome does not appear to have been commonly recognized (Dietzch and Wunderlich, 1969), though there are many references to more localized cartilage deficiencies in different parts of the bronchial tree (Emery, 1969; Lynch, 1970).

The purpose of this paper is to report a further 11

Received 12 November 1971.

*Nomenclature: bronchial generation after Weibel (1963), for instance trachea $=0$, and main bronchi $=1$. patients, to give a more comprehensive picture of the clinical features and natural history of the disorder, and to elucidate the disturbance in pulmonary function.

\section{Material and Methods}

Sixteen patients have been studied clinically and radiologically, and 12 of them have been followed up for periods between 2 and 16 years. 4 of the patients have died, but in only one was a necropsy and detailed histopathological studies carried out. One other patient with unilateral disease had a pneumonectomy, and study of his lung showed similar changes.

In 5 patients respiratory function studies were done. Lung volumes and maximum expiratory flow volume curves were measured using a flow displacement body plethysmograph (J. Mead, personal communication, 1970). Inspiratory resistance was measured by the oscillatory technique, using a method similar to that described by Goldman et al. (1970). Static elastic recoil curves were recorded in 4 patients using the method described by Turner, Mead, and Wohl (1968). The effect of respiratory frequency on dynamic compliance was measured in 4 patients (Woolcock, Vincent, and Macklem, 1969). 
Vital capacity and forced expiratory volume in one second VC and $\mathrm{FEV}_{1.0}$ were also measured using a Goddard Expirograph.

\section{Clinical Features}

All patients were born after an uncomplicated pregnancy and confinement, and no unusual features were detected during the neonatal period. No family history of a similar condition was elicited. There was no evidence of tuberculous infection, cystic fibrosis, or immunoglobulin deficiencies in any child. 8 of the 16 patients were boys. The initial symptoms developed in the first year of life in 13 patients, and in the remaining 3 before 3 years of age. In 11 patients the illness started with a cold, which was followed by a cough and wheezy breathing. In 5 patients the onset was acute, 2 had measles, 2 had pneumonia, and 1 had acute bronchiolitis. In these patients who were seriously ill, cough, tachypnoea, laboured breathing, a loud wheeze, and general malaise persisted after the more acute signs of the illness abated.

The continuing clinical pattern of illness was fairly uniform, though some patients were more severely affected than others (Table I). All had persistent cough and variable wheezing, so that a mistaken diagnosis of asthma was often made, and in a few a diagnosis of

\section{TABLE I}

\section{Symptoms in 16 Patients}

\begin{tabular}{l|l}
\hline Cough & 16 \\
Wheezing & 16 \\
Recurrent respiratory infections & 16 \\
Breathlessness & 13 \\
\hline
\end{tabular}

cystic fibrosis. The cough and wheeze were often aggravated by intercurrent respiratory tract infections, especially when the children were younger. During these acute exacerbations some of the children were very ill with fever, malaise, and breathlessness. Secondary bacterial infection frequently supervened, and the child was able to cough up mucopus. However, during adolescence respiratory infections became less frequent and cough and wheezing less persistent.

It was noted that all patients were much less active than normal children, and some became quite breathless on walking. After the age of approximately 8 to 10 years these children complained of breathlessness and said that they tired very easily. The oldest patient is just able to manage her housework, but finds she must rest frequently.

\section{Physical Signs}

Thirteen of the children were small, growth being on or below the 10th centile. 11 of the children had barrel-shaped chests, the result of persistent pulmonary hyperinflation and airways obstruction. 7 of them also had a marked pigeon-shaped deformity (Fig. 1).

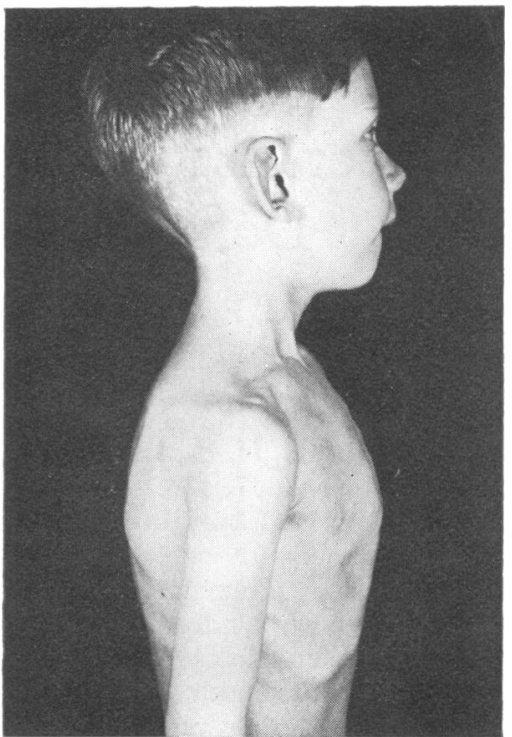

Fig. 1.-Case 1 illustrating the rib cage deformity.

Finger and toe clubbing were present in 11. All patients had a soft inspiratory wheeze and a louder prolonged expiratory wheeze; this was constantly present in some, and intermittently in others.

Widespread crepitations at the end of inspiration were another constant finding. These crepitations usually persisted for years, but in older children tended to disappear, but would reappear after a respiratory tract infection (Table II).

Sputum was coughed up by all the older children, but the quantity varied considerably. In some there were periods during which the cough was nonproductive. The cough in most patients was weak and not very effective owing to inability to expel a large volume of air quickly, due to collapse of the bronchial tree.

TABLE II

Physical Signs in 16 Patients

\begin{tabular}{l|l}
\hline Widespread crepitations & 16 \\
Growth retardation & 13 \\
Clubbing & 11 \\
Pulmonary hyperinflation & 10 \\
\hline
\end{tabular}

\section{Radiological Changes}

In the initial phase of the disorder there were increased hilar shadows and bronchovascular markings; a few of the more severely affected patients had varying degrees of pulmonary hyperinflation.

The signs of the well-established disease were gross pulmonary hyperinflation and in some patients dilated bronchi could be seen outlined as cylindrical air cavities 


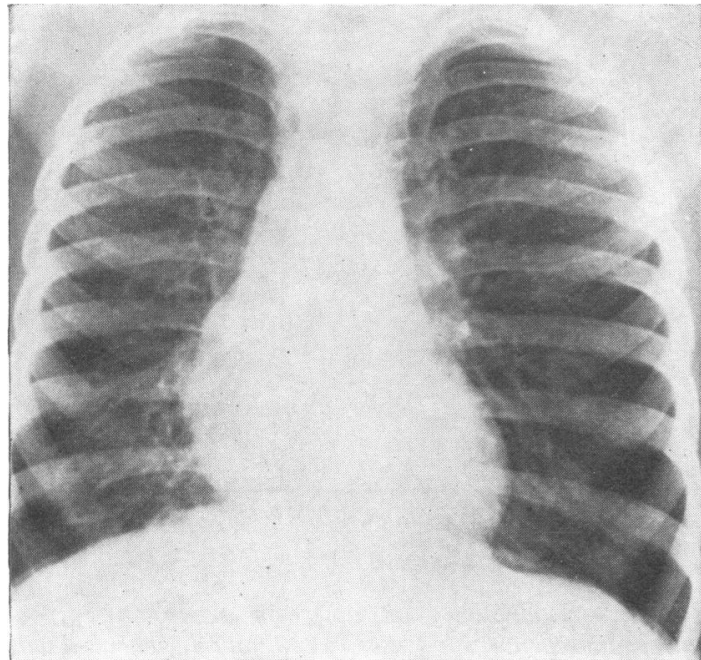

FIG. 2.-Case 1: chest radiograph showing dilated bronchi as cylindrical air cavities.

(Fig. 2). Persistent segmental or lobar collapse developed in 6 patients.

On bronchography the most characteristic findings were alternating ballooning and collapse of the dyeoutlined bronchi from the 3rd or 4th generation to the 7th or 8th generation during inspiration and expiration. These changes affected the entire bronchial tree in most patients, but in 5, some segments were only partially involved (Fig. $3 \mathrm{~A}$ and $\mathrm{B}$ ). In 1 patient the disorder was confined to the entire right lung, the left being

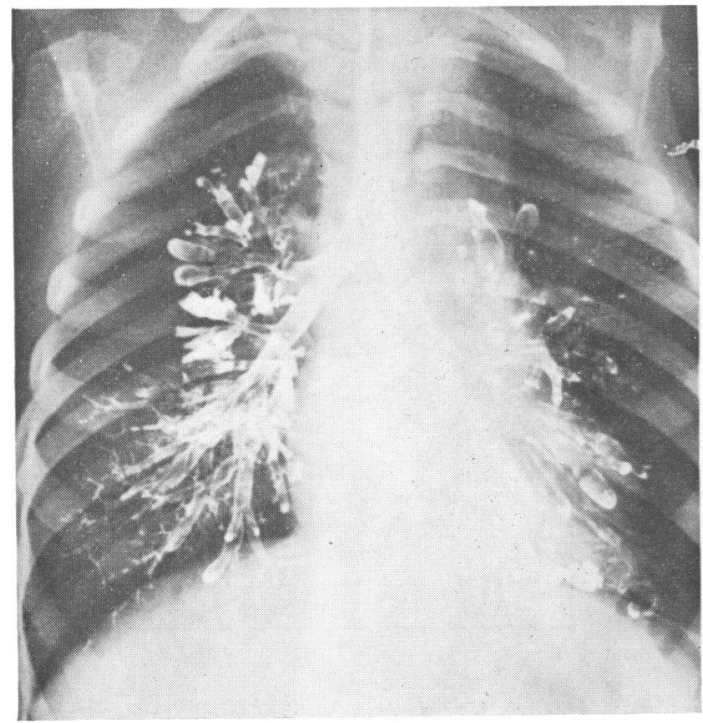

A normal. It was observed that the oily medium (Lipiodol) did not fill the smaller branches of the bronchial tree, and that remnants of the oil usually remained in the dilated bronchi for several days, even up to 1 week.

\section{Respiratory Function Studies}

Detailed results of the respiratory function studies are given in Table III. Fig. 4 is a maximum expiratory

\section{TABLE III}

Results of Respiratory Function Studies in 5 Patients with Bronchomalacia. Results are Expressed as Percentage of Predicted Normal, Except for RV/TLC and $F E V_{1} / V C$ Ratios Which are Expressed as Percentages

\begin{tabular}{l|r|r|r|r|r}
\hline \multicolumn{1}{c|}{ Case No. } & 1 & 2 & 3 & 4 & 5 \\
\hline Age & 10 & 18 & 22 & 16 & 10 \\
Sex & M & F & F & F & F \\
Height (cm) & 139 & 159 & 165 & 156 & 139 \\
Weight (kg) & 26 & 46 & 52 & 49 & 26 \\
RV & 260 & 295 & 405 & 305 & 171 \\
FRC & 137 & 165 & 205 & 159 & 107 \\
TLC & 108 & 116 & 132 & 120 & 83 \\
RV/TLC & 57 & 65 & 75 & 62 & 50 \\
VC & 72 & 46 & 49 & 63 & 60 \\
FEV & 40 & 33 & 23 & 36 & 46 \\
FEV & 60 & 51 & 36 & 54 & 43 \\
GInsp & 67 & 67 & 50 & 83 & 67 \\
V75VC & 16 & 26 & 18 & 16 & 26 \\
V50VC & 18 & 11 & 10 & 11 & 20 \\
V25VC & 6 & 11 & 9 & 7 & 15 \\
& & & & & \\
\hline
\end{tabular}

Note: $\mathrm{RV}=$ residual volume $; \mathrm{FRC}=$ functional residual volume; TLC = total lung capacity; VC = vital capacity; FEV = forced expiratory volume in one second; GInsp = inspiratory conductance; V75VC = maximum expiratory flow at $75 \%$ VC.

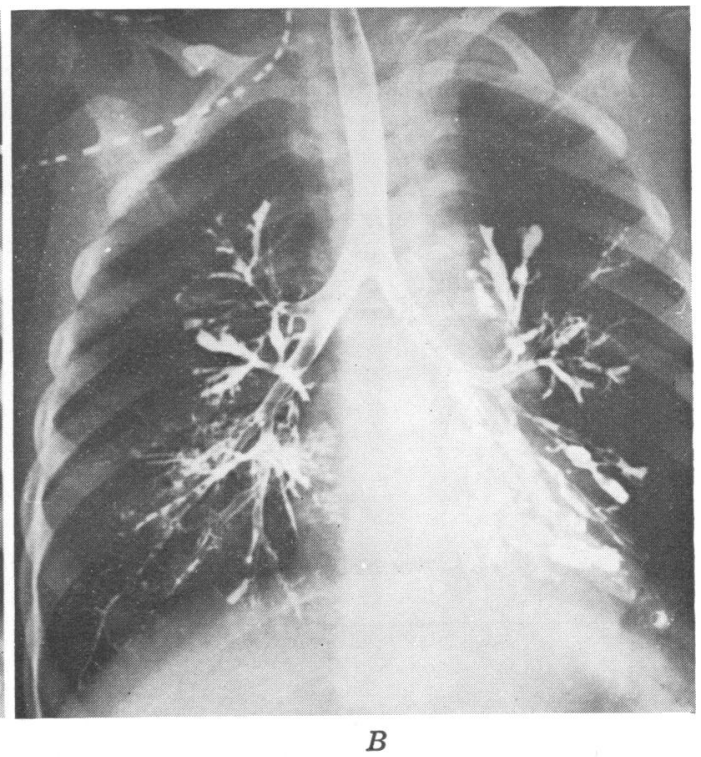

$B$ 
flow volume (MEFV) curve from Case 2, together with a curve from a normal patient of similar age. The static elastic recoil curves from 4 patients are shown in
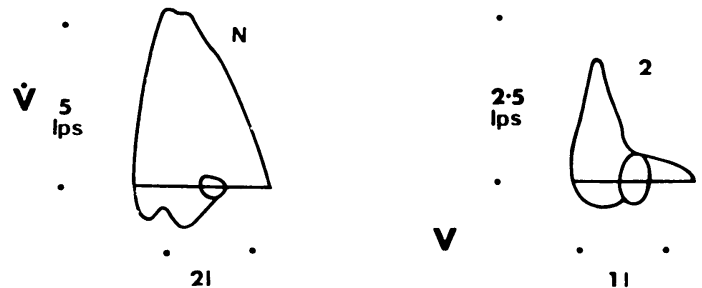

FIG. 4.-MEFV curves of normal subject and of Case 2. $\mathbf{V}$ represents volume; $\dot{\mathbf{V}}$ represents flow and scales shown. The tidal breathing pattern is also shown.

Fig. 5, lung volumes being expressed as percentages of measured total lung capacity (TLC). A curve for normal subjects showing the mean and $2 \mathrm{SDs}$ to the left of the mean is also shown (data of Turner et al., 1968, and M. E. Wohl and J. Mead, personal communication, 1970). In Fig. 6 the conductance of the upstream segment $\left(G_{u s}\right)$ (Mead et al., 1967) is shown, together with the conductance of the upstream segment for normal adolescents. The effect of frequency on dynamic compliance is shown in Fig. 7. No improvement in expiratory flow rate is shown in Fig. 7. No improvement in expiratory flow rate was observed after the inhalation of isoprenaline.

These results show gross hyperinflation of the lungs, and though the elastic recoil was reduced, it was not to the same degree as seen in adult patients with emphysema (Finucane and Colebatch, 1969), nor was the static compliance increased. There was considerable impairment of maximum expiratory flows at all lung volumes, and flow limitation started high in the vital capacity, suggesting rapid development of dynamic

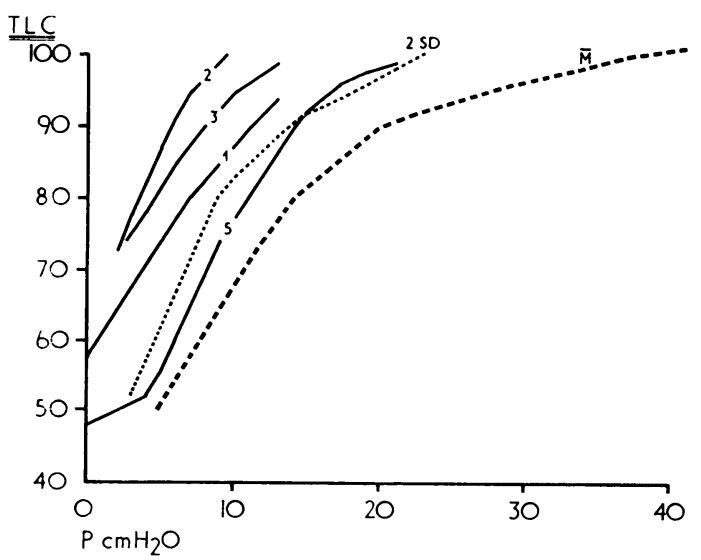

Fig. 5.-Static elastic recoil curves in Cases 1, 2, 3, and 5. Curves for the mean $(\bar{M})$ and for $2 S D$ to the left of the mean for normal children are shown as broken lines.

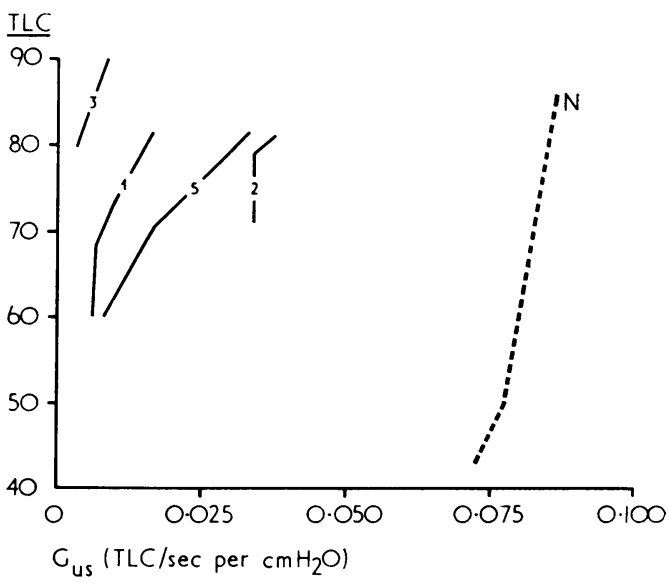

FIG. 6.-Conductance of upstream segment $\left(G_{u s}\right)$ at various lung volumes in Cases 1, 2, 3, and 5, compared with that for normal children and adolescents $(N)$.

compression of the larger airways. The reduction in maximum expiratory flows was most obvious at low lung volumes. Adult patients with severe chronic obstructive lung disease show a similar reduction on maximum expiratory flows but without the early evidence of dynamic compression (Takishima et al., 1967). It will be noted that the expiratory tidal flow in Case 2 was on the maximum expiratory flow volume curve (Fig. 4), and this phenomenon was seen in all 5 patients studied. This finding means that the patients could only increase their ventilation by breathing at a higher lung volume. It is thought that the phenomenon of tidal breathing on a flow volume curve is a major factor limiting ventilation in chronic obstructive lung disease (Takishima et al., 1967).

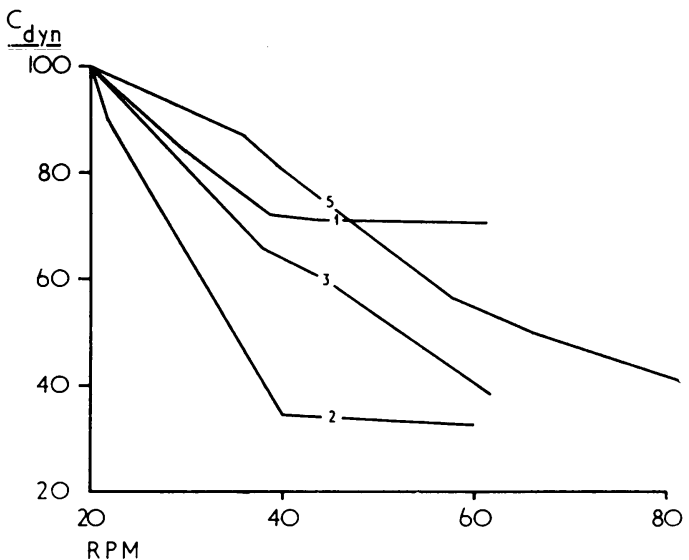

FIG. 7.-Dynamic compliance $\left(C_{d y n}\right)$ at frequencies of 20, 40, 60, and 80 breaths per minute in Cases 1, 2, 3, and 5 . 
Respiratory conductance was decreased in all patients but only in Case 2 was this significant. The scatter for normal values is quite wide. However, the conductance of the upstream segment was much decreased in all patients.

Dynamic compliance was frequency dependent in all subjects. This is indicative of maldistribution of ventilation. The cause of this can be regional variation in either compliance or airways conductance. The probability is that changes in the smaller airways were responsible for the maldistribution, particularly as respiratory conductance was only moderately abnormal.

\section{Natural History of Disorder}

Four of the 16 patients died, all before 5 years of age, 2 from respiratory failure after the development of pulmonary hypertension and gross cardiac enlargement, and 2 with pulmonary suppuration and respiratory failure. One patient was lost to follow-up. Of the remaining 12 patients, 8 are now aged between 15 and 22 years, and 4 between 3 and 9 years.

All these patients are limited in physical activity, 5 becoming breathless on mild exertion, e.g. walking on level ground for several hundred yards, and all but 3 are stunted in growth. When the patients were young frequent respiratory tract infections caused exacerbations of their symptoms and much ill health, but with increasing age these exacerbations became fewer.

\section{Management}

Attention has been directed towards controlling secondary infection by the use of antibiotics and clearing of secretions from the bronchial tree by encouraging coughing during breathing exercises and chest percussion. No specific bacteria were constantly cultured from their sputum. Haemophilus influenzae was the organism most commonly isolated, but pneumococci and staphylococci were also cultured from the same patient at different times.

\section{Discussion}

The uniform clinical and radiological findings in these patients and the pathological findings in 2 patients are very suggestive of a common underlying pathology. The radiological observations of ballooning and collapse of the bronchial tree from the region of the 3rd and 4th bronchial generation to the 6th or 8th, with changes of intrathoracic pressure during quiet tidal respiration, strongly suggested that the bronchi were unusually compliant.

Pathological studies in 2 patients have shown that the cartilage in the bronchi is deficient quantitatively, but not qualitatively, the cartilage plates being reduced in number, size, and thickness. The deficiency is most probably of congenital aetiology as there are no associated destructive or chronic inflammatory changes which would indicate that the cartilage had been destroyed consequent to inflammation. The clinical history of insidious onset of symptoms in infancy in 11 of the patients after a mild respiratory infection supports a congenital rather than acquired aetiology. The clinical features and pathology of the disorder do not resemble those which occur in severe adenovirus infections in which extensive bronchiectasis may occur (Lang et al., 1969; Becroft, 1971).

The gross pulmonary hyperinflation in most children, the persistent widespread crepitations over the chest, the prolonged forced expiration, and the raised intrathoracic pressure during expiration are all suggestive of extensive small airways pathology. The pathological studies in 2 patients showed extensive chronic inflammatory changes in the bronchiolar tree.

The respiratory function studies in the 5 severely affected patients were remarkably uniform, and this pattern of change has not been reported previously in any group of patients in this age range. These changes are gross pulmonary hyperinflation with exceptionally large residual lung volumes; poor static elastic recoil; severe impairment of maximum expiratory flow rates especially at low lung volumes; conspicuous decrease in conductance in the upstream segment but no decrease in inspiratory conductance (except for a mild reduction in one patient); and dynamic compliance which is frequency dependent.

These changes are in keeping with severe extensive small airways obstruction (Macklem and Mead, 1967), with collapse of the large airways during expiration and obstruction of air flow. The reduced elastic recoil could be secondary to long-standing pulmonary hyperinflation, or due to reduction in the elastic tissue in the lung, associated with a developmental anomaly. Quantitative estimations of the elastic tissue have not been made, and elucidation of this point must await further pathological studies.

While the aetiology and pathology of this disorder is not completely understood, the following is the probable sequence of events. It is postulated that these children were born with deficient cartilage in the bronchial tree. In infancy viral respiratory infection resulted in small airways obstruction which was slow to resolve by reason of a defective cough mechanism. The highly compliant bronchi would collapse during coughing, with limitation of the linear velocity of airflow (Mead et al., 1967). 
Poor drainage resulted in progressive obstructive small airways disease, pulmonary hyperinflation followed the small airways obstruction, and in 6 patients segmental or lobar collapse occurred. Impairment of ventilation developed owing to progressive airways obstruction and destruction of many of these smaller airways due to chronic inflammatory changes. Maldistribution of ventilation was a consequence of these changes.

The cause of the cartilage deficiency is uncertain. There is no familial incidence of the disorder and no observable deficits in cartilage outside the respiratory tract. It is uncertain whether other structures in the developing lung, such as elastic tissue, are involved. Localized areas of deficient cartilage of developmental aetiology in the trachea and bronchial tree have been recorded in infants by a number of workers (Gupta et al., 1968; Emery, 1969; Lynch, 1970). Varied clinical patterns of illness result, according to which part of the airways is involved.

The relation of the disorder described in this paper to tracheomalacia, bronchomalacia, and congenital lobar emphysema is of considerable interest. Campbell (1969), in a pathological study of 12 infants with congenital lobar emphysema, showed that there was a significant deficiency in the cartilage in all specimens. Some of these patients were 2 and 3 years of age at the time of removal of the affected lobe, and this suggests the possibility that MacLeod's syndrome (1954) may be a further variant of a developmental deficiency of cartilage in the bronchial tree.

It is probable that there are a number of uncommon lung disorders in which the basis is developmental deficiency of cartilage and possibly elastic tissue, in either a localized region or throughout a considerable part of the bronchial tree. Careful pathological studies to show quantitatively, cartilage and elastic tissue, are essential for further elucidation.

\section{Raprarances}

Becroft, D. M. O. (1971). Bronchiolitis obliterans, bronchiectasis, and other sequelae of adenovirus type 21 infection in young children. Fournal of Clinical Pathology, 24, 72

Campbell, P. E. (1969). Congenital lobar emphysema. Etiological studies. Australian Paediatric fournal, 5, 226.

Dietzch, H. J., and Wunderlich, P. (1969). Das Williams-CampbellSyndrom: eine sonderform generalisierter angeborener Bronchiektasen. Zeitschrift für Erkrankungen der Atmungsorgane, $130,387$.

Emery, J. L. (1969). The Anatomy of the Developing Lung, p. 74. Heinemann, London.

Finucane, K. E., and Colebatch, H. J. H. (1969). Elastic behavior of the lung in patients with airways obstruction. Fournal of Applied Physiology, 26, 330.

Goldman, M., Knudson, R. J., Mead, J., Peterson, N., Schwaber, J. R., and Wohl, M. E. (1970). A simplified measurement of respiratory resistance by forced oscillation. Fournal of Applied Physiology, 28, 113.

Gupta, T. G. C. M., Goldberg, S. J., Lewis, E., and Fonkalsrud, E. W. (1968). Congenital bronchomalacia. American fournal of Diseases of Children, 115, 88.

Lang, W. R., Howden, C. W., Laws, J., and Burton, J. F. (1969). Bronchopneumonia with serious sequelae in children with evidence of adenovirus type 21 infection. British Medical Fournal, 1, 73.

Lynch, J. I. (1970). Bronchomalacia in children. Clinical Pediatrics, 9, 279.

Macklem, P. T., and Mead, J. (1967). Resistance of central and peripheral airways measured by a retrograde catheter. fournal of Applied Physiology, 22, 395.

MacLeod, W. M. (1954). Abnormal transradiancy of one lung. Thorax, $9,147$.

Mead, J., Turner, J. M., Macklem, P. T., and Little, J. B. (1967). Significance of the relationship between lung recoil and maximum expiratory flow. Fournal of Applied Physiology, 22, 95.

Takishima, T., Grimby, G., Graham, W., Knudson, R., Macklem, P. T., and Mead, J. (1967). Flow volume curves during quiet breathing, maximum voluntary ventilation and forced vital capacities in patients with obstructive lung disease. Scandinavian Fournal of Respiratory Disease, 48, 384.

Turner, J. M., Mead, J., and Wohl, M. E. (1968). Elasticity of human lungs in relation to age. Fournal of Applied Physiology, 25, 664 .

Weibel, E. R. (1963). Morphometry of the Human Lung. Academic Press, New York; Springer, Berlin.

Williams, H., and Campbell, P. (1960). Generalised bronchiectasis associated with deficiency of cartilage in the bronchial tree. Archives of Disease in Childhood, 35, 182.

Woolcock, A. J., Vincent, N. J., and Macklem, P. T. (1969). Frequency dependence of compliance as a test for obstruction in the small airways. Fournal of Clinical Investigation, 48, 1097

Correspondence to Dr. H. E. Williams, Clinical Research Unit, Royal Children's Hospital Research Foundation, Royal Children's Hospital, Melbourne, 3052, Australia. 